\title{
The establishment of the fertile fish lineages derived from distant hybridization by overcoming the reproductive barriers
}

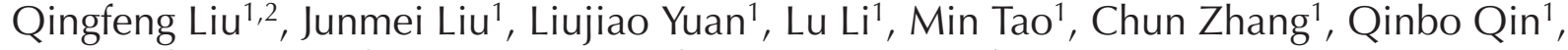 \\ Bo Chen ${ }^{2}$, Ming Ma², Chenchen Tang ${ }^{1}$ and Shaojun Liu ${ }^{1}$ \\ ${ }^{1}$ State Key Laboratory of Developmental Biology of Freshwater Fish, Engineering Research Center of Polyploid Fish \\ Reproduction and Breeding of the State Education Ministry, College of Life Sciences, Hunan Normal University, \\ Changsha, Hunan, People's Republic of China and ${ }^{2}$ Key Laboratory of Chemical Biology \& Traditional Chinese \\ Medicine Research, Ministry of Education, Hunan Normal University, Changsha, Hunan, People's Republic of China
}

Correspondence should be addressed to S Liu; Email: Isj@hunnu.edu.cn

\begin{abstract}
Distant hybridization refers to the cross between two different species or higher-ranking taxa. It is very significant if the new lineages with genetic variation, fertile ability, and improved characteristics can be established through distant hybridization. However, reproductive barriers are key limitations that must be overcome to establish fertile lineages derived from distant hybridization. In the present review, we discussed how distant hybridization is an important way to form new species by overcoming reproductive barriers and summarized effective measures to overcome reproductive barriers in order to create fertile lineages of fish distant hybridization. In addition, we described the utilization of the fish lineages derived from distant hybridization. Finally, we discussed the relationship between distant hybridization and Mendel's laws, which generally apply to the inbred hybridization. We aim to provide a comprehensive reference for the establishment of fertile fish lineages by overcoming reproductive barriers and to emphasize the significance of fish distant hybridization in the fields of evolutionary biology, reproductive biology, and genetic breeding.

Reproduction (2020) 159 R237-R249
\end{abstract}

\section{Introduction}

Distant hybridization, which is defined as the cross between two different species or higher-ranking taxa, can combine two different genomes, break through boundaries between species, and lay the foundation for genetic exchange between different species. Distant hybridization can also combine the biological characteristics of existing species, leading to changes in the phenotypes and genotypes of the progenies $\{$ Liu $2010\}$. With regard to phenotypes, distant hybridization can integrate advantages from both parents so that the offspring show heterosis in growth rate, fertility, and survival rate. With regard to genotypes, distant hybridization can change the number of chromosomes in progeny, causing diploidy, triploidy, and tetraploidy and, at the DNA level, it also leads to mutation and recombination in offspring (Liu 2010, Song et al. 2012). The formation of fertile lineages derived from distant hybridization can create new species variants and even new species.

Extensive researches have been made on the distant hybridization of major crops plants, such as corn, rice, soybean, wheat, cotton, and oilseed rape, and many excellent crop varieties have been obtained and popularized in large areas. For instance, the distant hybridization of common wheat (Triticum aestivum) and rye (Secale cereale) had resulted in the creation of a new crop species, octoploid triticale (Chen et al. 2018). In the animal kingdom, according to classical records, the mule of the post-Wei period in China, the yak of the Tang Dynasty, and the hybrid silkworms of the Ming Dynasty were all obtained by distant hybridization. Genner created the world's first recorded hybrid fish via the hybridization between carp (Cyprinus carpio) and goldfish (Carassius auratus) in 1558 (Wang et al. 2019a). From 1558 to 1980,1080 species in 56 families were used in fish hybrid breeding experiments and 3759 related research papers were published (Chen et al. 2018). Currently, hybridization is the most widely used technique in fish genetic breeding.

Reproductive barriers are key limitations that must be overcome to establish fertile lineages by distant hybridization. Reproductive isolation refers to the situation in which closely related species cannot mate with each other or cannot produce progeny or cannot produce fertile offspring. Reproductive isolation decreases the fitness of hybrid progeny, consistent with the idea that natural selection tends to eliminate hybrids to ensure species independence. However, if the progeny encounter a suitable niche, 
the reproductive isolation mechanism may disappear or partially disappear, enabling the formation of a new species. Previous studies have indicated that, in nature, approximately $10 \%$ of animals and $25 \%$ of plants have undergone hybridization with at least one other species and more than $40 \%$ of angiosperms have undergone hybrid introgression (Chen et al. 2018). Thus, distant hybridization is one of the main driving forces of genomic evolution and seems to facilitate speciation (Mallet 2007). After reviewing the literature and longstanding research on fish distant hybridization from our laboratory, here we systematically described how to overcome reproductive isolation in order to establish fertile fish lineages through distant hybridization. We also provided useful materials and data for distant hybridization breeding.

\section{Distant hybridization and reproductive isolation}

A large number of breeding practices have indicated that it is difficult to achieve distant hybridization because of different degrees of reproductive isolation (Wolff \& Peters-van Rijn 1993, Deng et al. 2010a,b). With regard to distant hybridization of plants, failure of any process from the germination to growth during pollination and from the completion of double fertilization to the development of zygote will lead to hybridization failure. In the natural hybridization among animals, reproductive isolation is also related to the mating season, place, time, and pheromone secretion and any factor occurring out of order will directly result in hybridization failure (McClure \& Franklin-Tong 2006, Berger 2008). According to the time period, reproductive isolation of animals and plants can be classified as pre-zygotic or post-zygotic reproductive isolation.

\section{Pre-zygotic reproductive isolation is a segregation that occurs before fertilization and is usually a barrier under natural conditions}

Pre-zygotic reproductive isolation includes ecological and habitat isolation, seasonal isolation, behavioral isolation, mechanical isolation, and physiological isolation.

Ecological and habitat isolation is defined as a barrier caused by environmental changes such as crustal movement and climate change that prevent two species from achieving successful distant hybridization. For instance, throughout geological history, the formation of high mountains and increases in elevation geographically separated Pinus yunnanensis from Pinus densata, resulting in asynchronism of flowering, and this asynchronism hindered the exchange of genes between the two species and caused isolation (Song et al. 2002). In addition, it has been found that the different genetic structure of Atlantic cod (Gadus morhua) arose in the two major ocean front areas near Iceland due to factors such as ocean currents and differences in sea water temperature, sea depth, and ocean fronts that prevented the gene flow between the two groups (Pampoulie et al. 2006).

Seasonal isolation, also named as temporal isolation, arises from the tendency of organisms to have certain times and seasons of reproduction (mating periods in animals, flowering and pollination periods in plants, etc.). If the breeding seasons and times of close species are different, it will cause seasonal isolation, which prevents the exchange of genes and leads to failure of distant hybridization. For example, a study on Ophrys species (Op. iricolor and Op. mesaritica) revealed that the reproductive isolation of these plants is caused by different flowering seasons between the two species (Xu et al. 2012). Furthermore, Rana clamitans, Rana amurensis, and Rana pipiens live in the same ponds in North America; however, $R$. amurensis begins to spawn first, when the temperature of the water reaches $7^{\circ} \mathrm{C}$ (in February); R. pipiens spawns next, when the water temperature reaches $12^{\circ} \mathrm{C}$ (in early March); and $R$. clamitans starts to spawn last, when the water temperature reaches $16^{\circ} \mathrm{C}$ (at the end of March). These different spawning seasons cause the reproductive isolation among the three species that could otherwise be hybridized to produce offspring (Chen et al. 2018).

Behavioral isolation is a situation in which it is difficult for two or more closely related groups to mate with each other and produce progeny because of different mating behaviors. Behavioral isolation occurs mainly in the animal distant hybridization. For example, although they have the same flowering period and are distributed in the same area, Ophrys exaltata, Ophrys garganica, and Ophrys sphegodes cannot cross-pollinate, because the pollinators of the three species are three different insects (Sedeek et al. 2014). In addition, a study on the acoustic behavior of two species of Laupala in Hawaii revealed that differences in the strength and frequency of the sounds hindered normal mating between these species, resulting in reproductive isolation $(\mathrm{Xu} \&$ Shaw 2019).

Mechanical isolation, a classification of isolation proposed by Dobzhansky, refers to a phenomenon in which animals and plants cannot produce offspring due to the improper structure of the mating apparatus or the flower device. For example, Pedicularis L. can have different corolla types, and different species of drones use different access methods for pollination; these differences prevent pollen interference between different species in the same domain, resulting in mechanical isolation. However, Dobzhansky believed that the phenomenon of inability due to differences in reproductive organ structure between very closely species was much less than estimated (Sheppard 1971).

Physiological isolation is a phenomenon in which fertilization cannot be completed after a plant has been pollinated or after an animal has mated because 
of physiological incompatibility. In vivo, the gamete or gametophyte of one species cannot survive in another organism; in vitro, the male and female gametes may not attract each other. For example, when male rabbit (Oryctolagus cuniculus) sperm reach the oviducts $6 \mathrm{~h}$ after mating, female rabbits begin to ovulate; conspecific sperm can live for approximately $38 \mathrm{~h}$ in the female rabbit reproductive tract, while heterologous sperm can survive only $3-4 \mathrm{~h}$ in the unaccommodating environment, thus preventing normal fertilization (Chen et al. 2018). For fish distant hybridization, ecological isolation and seasonal isolation may be easier to overcome than other isolations. These two kinds of isolation may be solved through transportation and water temperature control.

\section{In post-zygotic reproductive isolation, the gametes of two species can undergo fertilization, but the hybrids cannot form stable populations for various reasons}

Causes of post-zygotic reproductive isolation include hybrid inviability, hybrid sterility, and hybrid weakness.

Hybrid inviability refers to a situation in which a zygote is formed but cannot survive, a situation in which a zygote can survive but the embryo dies after a certain period of development or a situation in which an embryo can develop into a larva but the larvae cannot survive to sexual maturity. For example, hybrid progeny of two strains of Arabidopsis thaliana, UK1-1 and UK-3, cannot survive due to abnormal activation of the immune system (Bomblies et al. 2007).

Hybrid sterility refers to a situation in which the hybrid progeny of different species form a zygote that can develop into a mature $F_{1}$ hybrid, but the hybrid could not produce offspring. For instance, the hybridization between donkeys (with 62 chromosomes) and horses (with 64 chromosomes) can produce surviving offspring with 63 chromosomes. However, the hybrid progeny cannot complete germ cell meiosis and therefore cannot develop normal gametes because of chromosome association disorder, resulting in infertility of the offspring (Sharma \& Gill 1983). Similarly, researchers who have conducted inter-species crosses between Argopecten irradians and Argopecten purpuratus have observed bidirectional fertilization and successful hatching for all crosses, without evidence of pre-zygotic isolation. Haematoxylin and eosin (HE) staining of paraffin slices of three gonads of mature hybrids has revealed that the male gonads are obviously degraded, lacking active sperm, while the female gonads are well developed with abundant normal-appearing eggs. However, the hybrid eggs cannot be backcrossed with the parental species, exhibiting typical post-zygotic hybrid sterility (Zhang et al. 2012).

Hybrid weakness refers to a situation in which interspecific hybridization can form fertile $F_{1}$ hybrids, but most of the recombinant genotypes of $\mathrm{F}_{2}$ or backcross progeny are not coordinated and thus cannot survive. For example, when female spotted mutants of Gossypium hirsutum and male Gossypium tomentosum are crossed, the $F_{1}$ offspring survive and are fertile. However, the $F_{2}$ hybrids obtained by $F_{1}$ self-crossing have small embryos and the seedlings cannot germinate leading to death. In addition, studies have shown that there is a hybrid decay mechanism in the recombinant genotype of $F_{2}$ organisms that causes them to succumb to the pressure of natural selection, failing to survive (Chen et al. 2018).

\section{Intrinsic and extrinsic reproductive isolation}

Recently, based on research on reproductive isolation mechanisms at the genome level, scientists have divided reproductive isolation into intrinsic reproductive isolation and extrinsic reproductive isolation (Seehausen et al. 2014). Extrinsic reproductive isolation is considered to rely on genotype interactions with the environment and is usually caused by ecological isolation, geographical isolation, and evolutionary divergence. In Australia, researchers have studied the ecological and genetic mechanisms underlying the blockage of gene flow, morphological maintenance, and genetic specificity between two adjacent populations of coastal groundsels (Senecio lautus). Extrinsic isolation mechanisms prevent gene communication, while intrinsic isolation mechanisms are weak in $\mathrm{F}_{1}$ hybrids (Melo et al. 2014). It is generally believed that pre-zygotic types of reproductive isolation, such as ecological isolation and behavioral isolation, are more likely to be related to extrinsic reproductive isolation. Intrinsic reproductive isolation is usually caused by genomic conflicts, and it is generally independent of the external environment. Researchers have found that multiple genetic factors might be involved in intrinsic reproductive isolation, such as speciation genes, chromosomal mechanism variations, Bateson-Dobzhansky-Muller incompatibilities, and genetic partial separation (Wu \& Ting 2004, Rieseberg \& Willis 2007).

\section{Scholars have also identified three main reasons for distant hybridization incompatibility}

Differences in chromosome number or karyotype between parents that are too large

Differences in the numbers of chromosomes between parents result in inconsistencies in the alleles of chromosomes from the parents during zygote formation and disorder of gene regulation prevents normal embryonic development, eventually causing the hybrid individuals to fail to develop or even to die. Most fish distant progenies cannot survive or those who have low survival rates might belong to this case. For example, in the distant hybridization between grass carp (Ctenopharyngodon idellus) and carp, the carp had 50 pairs of chromosomes, while the grass carp had 
only 24 pairs of chromosomes. The carp and grass carp do not have corresponding alleles at many gene loci, resulting in the hybrid combination rarely producing viable diploid hybrid progenies (Ye et al. 1989). The parents of some hybrid combinations have the same numbers of chromosomes but different karyotypes, which also causes disorder of some allele combinations; the parent species Sinilabeo decorus tungting $(2 n=50)$ and Cirrhinus molitorella $(2 n=50)$ are relevant examples (Wang et al. 1986). It is generally believed that the closer the karyotype between parents, the easier to achieve fish distant hybridization. Therefore, the studies on karyotypes and chromosome numbers are important for the analysis and prediction of fish species for distant hybridization.

\section{Differences in the temporal order of gene locus activation or enzyme expression}

As the degree of fish evolution increases, the genetic locus of enzymes also increase; isozymes with a shorter history of differentiation have stronger temporal limitations. When the genetic relationship between hybrid parents is far, the temporal order of parental allele expression may be out of sync or mutually inhibitory. Enzyme incompatibility can lead to spatiotemporal disorders in the induction of hybrid embryonic tissue and organ formation, thus resulting in the production of deformed offspring or offspring that cannot survive. For instance, a study on isozymes in hybrid progenies of grass carp (\$) and blunt snout bream (Megalobrama amblycephala, of found that, during the development of diploid hybrids, malate dehydrogenase $(\mathrm{MDH})$ and glucose-6-phosphate dehydrogenase $(\mathrm{G} 6 \mathrm{PDH})$ are expressed as a single expression (in females), which reduces the activity of the enzymes; the expression times of other sites with partial affinity (such as esterase and lactate dehydrogenase) are also changed, resulting in abnormal development or even death of the diploid hybrids.

\section{Nuclear-cytoplasmic incompatibility}

The maternal egg cytoplasm controls the speed of hybrid embryo gene expression. When the cytoplasm of an egg is incompatible with the nuclear DNA of a sperm cell, gene expression can be blocked or accelerated, thus causing the embryo to develop abnormally until death. For instance, the embryos of crosses between grass carp () and triangular bream (Megalobrama terminalis, ơ) can develop normally, and $80-90 \%$ of the hybrid fry can be obtained. However, when triangular bream is used as female and grass carp is used as male, the embryos of the offspring are difficult to survive. These results show that the reason why the progenies of reverse cross combination cannot develop normally is not the incompatibility between the parental nuclear DNA, but rather to the abnormal coordination of the nuclear DNA of triangular bream with the sperm of grass carp (Liu 1993). This phenomenon also occurs in the cross of common carp (Cyprinus carpio) with C. auratus and of green sunfish (Lepomis cyanellus) with largemouth bass (Micropterus salmoides) (Whitt 1981, Liu 1993).

\section{Distant hybridization and species formation}

Since Darwin's point of view regarding species origin surfaced, species formation has become one of the most active areas in evolutionary biology research. In particular, the rapid development of molecular population genetics, bioinformatics, and genomics in the past two decades has made the study of species formation become a popular research topic in the field of evolutionary biology. It is generally believed that new species often form gradually through differentiation from a single species; however, another way of new species formation is through hybridization. The simultaneous contribution of two distinct species to form a new species through genetic hybridization (Mavárez et al. 2006), and this hybridization is the distant hybridization. Thus, distant hybridization is one of the main driving forces of genomic evolution and seems to facilitate speciation (Mallet 2007). However, it is necessary for successful distant hybridization to overcome reproductive isolations. Reproductive isolation is an important attribute for the definition of modern species, and it is a key factor for the independent and stable coexistence of various species in nature. So, reproductive isolation has played a protective role during the biological evolution of existing species. Speciation through hybridization can be divided into three categories according to whether the number of chromosomes doubles and whether there is reproductive isolation between the offspring and the parents: polyploidization, homoploid hybrid speciation, and introgressive hybridization.

\section{Polyploidization is a widely recognized method of species formation}

Paired chromosomes from different species cannot properly separate during meiosis, resulting in infertility in the hybrid progeny. However, in polyploidy (such as tetraploid species), by chromosome doubling, each paired chromosome contains two sets of chromosomes from one parent during meiosis, and the normal separation can be guaranteed, thereby the fertile hybrid offspring can be obtained. And, reproductive isolation can be established because the chromosomes are of polyploidy hybrid progeny and the parents do not match. Thus, polyploid hybrid offspring can be relatively easily identified as a new species. Distant hybridization is an important way to produce polyploid hybrid. For example, small numbers of germ cells can produce unreduced gametes in the $\mathrm{F}_{1}$ hybrids of Raphanus 
sativus and Brassica oleracea, and the allotetraploid Raphanobrassica can be obtained in $F_{2}$ by $F_{1}$ selfcrossing (Karpechenko 1927). In the hybridization of red crucian carp (C. auratus red var., o) and common carp $\left({ }^{\star}\right)$, the females and males of $F_{2}$ hybrids are able to produce diploid eggs and diploid spermatozoa that can undergo fertilization to form allotetraploid fish (Liu et al. 2001).

\section{Homoploid hybrid species}

Homoploid hybrid species formation refers to the hybridization of two species with the same chromosome number, and the hybrid individuals eventually form a new species that is different from either parent species. Compared with the formation of polyploidy hybrid species, the formation of homoploid hybrid species is more difficult, mainly in two aspects. First, once allopolyploid hybrid species are produced, the hybrids of allopolyploids and their parents are not able to produce germ cells because normal chromosome pairing cannot be completed; thus, the reproductive isolation is established. Correspondingly, homoploid hybrid species do not immediately exhibit reproductive isolation from their parental species (Soltis \& Soltis 2009). Second, because the genomes of allopolyploid hybrid species include the genetic components of two complete parental species, the integrity of the genomes is effectively guaranteed; however, the genomes of homoploid hybrid species lack this protective mechanism. The most classic case of homoploid hybrid formation was revealed in a study on the genus Helianthus in which hybridization between $H$. annuus and $H$. petiolaris produced three homoploid hybrids with significant differences in morphology and habitat. Further studies also revealed that these three new species exhibit different superparental traits and can adapt to extreme environments (Gross et al. 2003).

\section{Introgressive hybridization}

Introgressive hybridization was proposed by Anderson and Hubricht through the study of plant hybridization (Anderson \& Hubricht 1938). It refers to repeated backcrossing between the hybrid offspring and one or both parents eventually bring a parental trait to the other parent. Introgressive hybridization in plants has been widely reported and applied for breeding protection. Introgressive hybridization of animals is mainly found in fish, insects, amphibians, reptiles, birds, and mammals. Introgressive hybridization generally does not produce new species, but if the fertile progeny no longer backcross with the parental population or occupy a new environment, new hybrid species may be formed (Abbott 2003).

\section{Measures for overcoming reproductive barriers in fish distant hybridization}

There are more than 35,358 species of fishes; thus, there are abundant germplasm resources for fish distant hybridization. Since 1558, when Genner crossed carp and goldfish to obtain the world's first recorded hybrid fish (Chen et al. 2018), a large number of successful cases of fish distant hybridization have been reported (Wang et al. 2019a). According to literatures and to results from our team's research on fish distant hybridization, we introduced several measures for overcoming reproductive barriers:

\section{Exploration of the rules of fish inheritance and reproduction}

Genetic rules at the chromosome level

Both chromosome numbers and karyotypes from parents have crucial impacts on the success of distant hybridization. And, chromosome as the carrier of genetic material is an important basis for studying the genetic rules between progenies derived from distant hybridization and parents. We have conducted 37 distant crosses and obtained 30 hybrid combinations with surviving offspring in long-term and systematic studies on freshwater fish with 100 and 48 chromosomes (including red crucian carp, white crucian carp, common carp, grass carp, blunt snout bream, Bleeker's yellow tail, bighead carp, topmouth culter, and other commercial fishes). From the perspective of the chromosome numbers of the parents, we summarize the genetic rules:

When the number of the maternal chromosomes is equal or similar (no significant difference) to that of the paternal chromosomes, the allotetraploid lineages and allodiploid lineages can be established by overcoming the reproductive barriers (Fig. 1). For example, an allotetraploid lineage has been obtained from $F_{3}$ to $F_{26}$ derived from the crossing of red crucian carp $(2 n=100$, o) and common carp $\left(2 n=100, \delta^{\star}\right)$, because the $F_{2}$ hybrids produced unreduced gametes (Liu et al. 2001). An allodiploid lineage $\left(F_{1}-F_{6}\right)$ has been established by the hybridization of blunt snout bream $(2 n=48$, ㅇ) and topmouth culter $(2 \mathrm{n}=48,0$, Culter alburnus) (Xiao et al. 2014). In addition, the $F_{1}$ hybrids $(2 n=45)$ can be obtained by the hybridization of Channa maculate $(2 n=42, q) \times$ Channa argus $\left(2 n=48,0^{\star}\right)$, and the $F_{2}$ hybrids were produced by self-crossing of $\mathrm{F}_{1}$ (Liu et al. 2011). Furthermore, we obtained the $F_{1}$ hybrids $(2 n=49)$ by the hybridization of blunt snout bream $(2 n=48$, o) $\times$ Chinese rare minnow (Gobiocypris rarus, $2 \mathrm{n}=50$, $\left.{ }^{\star}\right)$, and the fertility of the $F_{1}$ hybrids needs further observation in the future.

When the number of the maternal chromosomes is larger than that of the paternal chromosomes, 


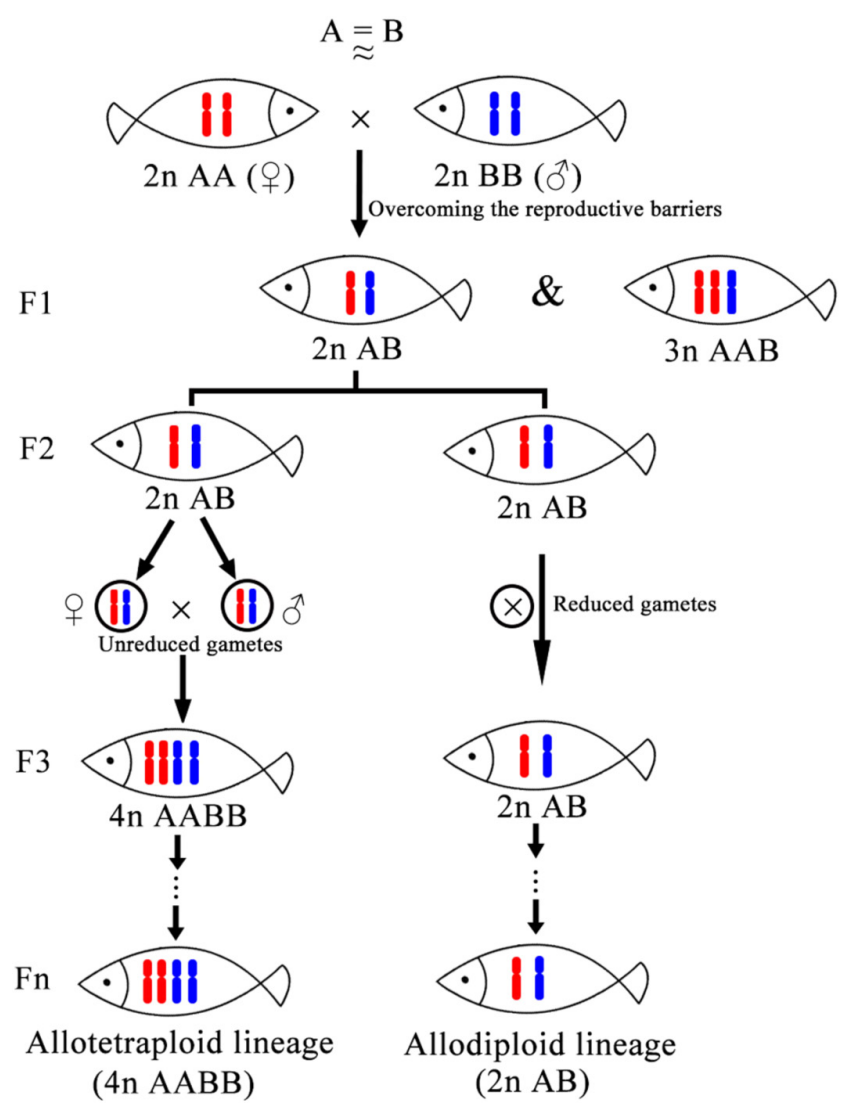

Figure 1 The fertile lineages were obtained when the number of parental chromosomes is equal. In this cross, allotetraploid and allodiploid fish lineages can be established by overcoming the reproductive barriers.

autotetraploid lineages and autodiploid lineages can be established by overcoming the reproductive barriers (Fig. 2). For instance, an autotetraploid lineage and an autodiploid lineage have been obtained from the crossing of red crucian carp $(2 n=100$, क $)$ and blunt snout bream $\left(2 \mathrm{n}=48, \delta^{\star}\right)$ (Qin et al. 2010, 2014b). Additionally, an autodiploid crucian carp-like lineage $\left(\mathrm{F}_{1}-\mathrm{F}_{6}\right)$ has been established by the hybridization of common carp (q, $2 n=100)$ and blunt snout bream $(0,2 n=48$ ) (Wang et al. 2017). Furthermore, an autodiploid red crucian carp-like lineage $\left(\mathrm{F}_{1}-\mathrm{F}_{4}\right)$ has been successfully obtained through hybridization of koi carp (Cyprinus carpio haematopterus) (ㅇ, $2 \mathrm{n}=100$ ) and blunt snout bream ( ${ }^{*}$, $2 n=48$ ); and, a new type of autodiploid goldfish-like lineage $\left(\mathrm{F}_{1}-\mathrm{F}_{3}\right)$ has been created by self-crossing of the red crucian carp-like fish (Wang et al. 2018).

When the number of the maternal chromosomes is fewer (significantly fewer) than that of the paternal chromosomes, the offspring are unlikely to survive. However, when the numbers of chromosomes in the parents are in a ploidy relationship, the progenies can survive even if the number of the maternal chromosomes is fewer (significantly fewer) than that of the paternal chromosomes. In this case, the coordination of the parents is good. For example, the triploid fish has been produced by the hybridization of the white crucian carp (C. cuvieri, $2 \mathrm{n}=100$, $\%$ ) and the allotetraploid hybrids $(4 n=200$, ơ) (Liu et al. 2001, Guo et al. 2006). Additionally, autotriploid fish has been produced by the crossing of red crucian carp $(2 n=100$, क $)$ and autotetraploid hybrids $\left(4 n=200,0^{*}\right)$, and allotriploid hybrids has been produced by the hybridization of common carp $(2 \mathrm{n}=100$, $\%)$ and autotetraploid hybrids $\left(4 n=200, o^{\star}\right)($ Hu et al. 2019).

\section{Nuclear-nuclear and nuclear-cytoplasmic compatibility}

The genetic rules at the chromosome level revealed the influence of parental chromosome number on distant hybridization. The degree of matching of the parental chromosome number will affect the nuclear-nuclear and nuclear-cytoplasmic compatibility in $\mathrm{F}_{1}$ hybrids (Table 1 ). Nuclear-nuclear and nuclear-cytoplasmic compatibility is closely related to the survival rate of $F_{1}$ hybrids derived from distant hybridization. In $\mathrm{F}_{1}$ hybrids, the nuclearnuclear and nuclear-cytoplasmic compatibilities include the compatibility between the maternal nuclear material (including the genome) and paternal nuclear material (including the genome), between the maternal nuclear material (including the genome) and the cytoplasm and between the paternal nuclear material (including the genome) and the cytoplasm.

When the number of maternal chromosomes is equal to that of paternal chromosomes, the maternal genomepaternal genome, maternal genome-cytoplasm, and

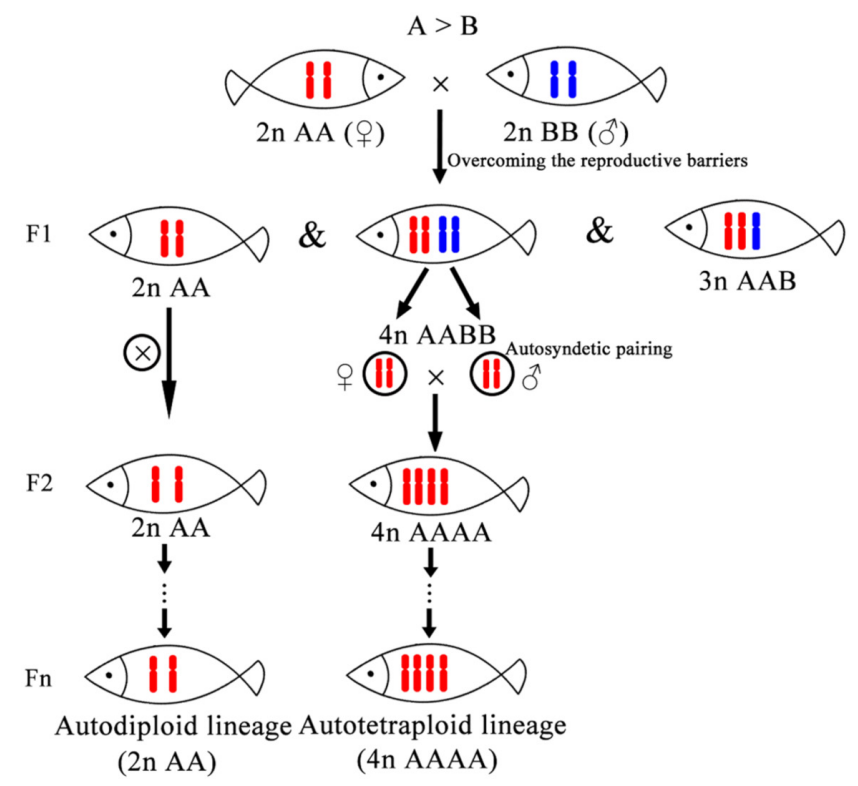

Figure 2 The fertile lineages were obtained when the number of maternal chromosomes is larger than that of paternal chromosomes. In this cross, autotetraploid and autodiploid fish lineages can be established by overcoming the reproduction barriers. 
Table 1 The number of chromosomes and the degree of nuclear-nuclear and nuclear-cytoplasmic compatibility in distant hybrid $\mathrm{F}_{1}$.

\begin{tabular}{|c|c|c|c|c|c|}
\hline \multirow{2}{*}{$\begin{array}{l}\text { Number of } \\
\text { parental } \\
\text { chromosomes }\end{array}$} & \multicolumn{3}{|c|}{ Compatibility } & \multirow[b]{2}{*}{$\begin{array}{l}\text { Overall } \\
\text { compatibility }\end{array}$} & \multirow[b]{2}{*}{ Survival rate } \\
\hline & Maternal nucleus - cytoplasm & Maternal nucleus - paternal nucleus & Paternal nucleus - cytoplasm & & \\
\hline$\phi<0^{*}$ & $\sqrt{ }$ & $\times$ & $x$ & poor & low \\
\hline$q=\sigma^{x}$ & $\sqrt{ } \sqrt{ }$ & $\sqrt{ } \sqrt{ }$ & $\sqrt{ } \sqrt{ }$ & good & good \\
\hline$q>0$ & $\sqrt{ } \sqrt{ }$ & $\sqrt{ }$ & $\sqrt{ }$ & normal & normal \\
\hline
\end{tabular}

$\times$ : poor compatibility, $\sqrt{ }$ : normal compatibility, $\sqrt{ } \sqrt{ }$ : good compatibility.

paternal genome-cytoplasm compatibility are good; in this case, $F_{1}$ hybrids have a good survival rate.

When the number of maternal chromosomes is more than that of paternal chromosomes, the maternal genetic material is dominant in $\mathrm{F}_{1}$ hybrids, and the hybrids have a certain developmental ability. In this case, the maternal genome-paternal genome, maternal genome-cytoplasm, and paternal genome-cytoplasm compatibility are better than the third case but not better than that in the first case, and the $F_{1}$ hybrids of this kind of cross can produce offspring with a certain survival rate.

When the number of maternal chromosomes is fewer (significantly fewer) than that of paternal chromosomes, the maternal genetic material is not dominant in $F_{1}$ hybrids, and the $F_{1}$ hybrids exhibit poor physiological development ability. The maternal genome-paternal genome, maternal genome-cytoplasm, and paternal genome-cytoplasm compatibility in $\mathrm{F}_{1}$ hybrids is poor, so the survival rate in this case is generally very low. However, when the numbers of chromosomes of the parents are in a ploidy relationship, the maternal genome-paternal genome, maternal genome-cytoplasm, and paternal genome-cytoplasm compatibility is good and the $F_{1}$ hybrids can survive.

\section{Genetic characteristics at the molecular level}

With the rapid development of sequencing technology, we have carried out a series of genomic and transcriptome sequencing studies on existing distant hybrid fish lineages. Studies have found that these fish lineages derived from distant hybridization have the same genetic characteristics and these fish lineages not only inherit the genetic material from the female parent, but also that from the male parent with different degrees.

For allodiploid lineages and allotetraploid lineages derived from distant hybridization, the progenies contain two subgenomes from the parents. For example, a high proportion of chimeric genes $(>9 \%)$ has been found among orthologous genes in the transcriptomes of allotetraploid fish (Liu et al. 2016), and the same results have been obtained in analyses of bacterial artificial chromosome (BAC) libraries of allotetraploid fish (Ye et al. 2017). Furthermore, we have compared the orthologous genes in the transcriptomes of hybrids (WR) from white crucian carp (Q) and red crucian carp ( $\sigma^{*}$ ) and have found $19.04 \%$ chimeric genes in $W R-F_{1}$ hybrids; we also found that some chimeric genes can be inherited from WR-F $F_{1}$ to $W R-F_{2}$ (Liu et al. 2018). Chimeric genes might have formed after the two genomes merged and before whole genome duplication that lead to tetraploidization (Rogers et al. 2009). The high frequency of chimeras also might result from largescale DNA repair via recombination or non-homologous end-joining, or even transposon activity (Wang et al. 2006, Levin \& Moran 2011, Bao \& Yan 2012, Fedoroff 2012). The allotetraploid fish and WR fish have four sets and two sets of genomes from their parents, respectively; at the DNA level, the genetic material from the parents is combined into one by the form of chimeric genes, which facilitates the re-diploidization of the fish lineages derived from distant hybridization and establishes the genetic basis for overcoming the reproductive barriers.

For autodiploid lineages and autotetraploid lineages derived from distant hybridization, the genomes of the progeny are dominated by the maternal genome and the paternal DNA fragments are inserted into them. For instance, we have identified DNA fragments from the original male in autotetraploid fish and autodiploid red crucian carp obtained through distant hybridization (Chen et al. 2018). These inserted DNA fragments also facilitate the re-diploidization of these lineages. This form of diploidization constitutes an important genetic basis for overcoming reproductive barriers.

\section{Reproductive rules of fish distant hybridization}

The tetraploid and diploid hybrid fish lineages established by distant hybridization are sexually fertile. Through long-term research, we have found that allodiploid fish $(2 \mathrm{n}=100)$ produce unreduced gametes, which is an important reason for the formation of allotetraploid fish lineages $(4 n=200)$; for instance, the $\mathrm{F}_{2}$ progenies from distant hybridization of red crucian carp (\$) and common carp ( $\left.0^{*}\right)$ can produce unreduced gametes, allotetraploid fish can be obtained in $F_{3}$ by $F_{2}$ self-crossing, and an allotetraploid lineage $\left(\mathrm{F}_{3}-\mathrm{F}_{26}\right)$ can be formed by continuous self-crossing (Liu et al. 2001). The homologous diploid gametes and homologous triploid gametes produced by allotetraploid are the key factors for the formation of autotetraploid lineages; for example, allotetraploid fish $(4 n=148)$ have been obtained in the $\mathrm{F}_{1}$ hybrids by the distant hybridization between red crucian carp and blunt snout bream, autodiploid gametes have been produced during the self-crossing process of allotetraploid fish $(4 n=148)$, and 
autotetraploid $(4 n=200)$ fish lineages have been formed (Qin et al. 2014b). Similarly, haploid gametes produced by diploid hybrid fish are the important causes of diploid fish lineages. However, although the reproductive barriers have been overcome and the progenies derived from distant hybridization have been successfully obtained, there are cases in which only a single sex of offspring is fertile. In this case, the fertile hybrids can be backcrossed with one of the parents; as the backcross generations increase, fertility will gradually improve until it is recovered.

\section{Promotion of fertilization}

Promotion of fertilization to overcome pre-zygotic reproductive isolation is of great significance for fish distant hybridization. The breeding time of the parents of hybrid fish can be prolonged to solve the problem of slow gonadal development (more than 1-2 years in normal parents). For parents who produce only a small number of water-like semen or small numbers of eggs, the semen and eggs can be gathered for artificial insemination. Drip-type constant-temperature incubation systems can be established to solve the problem of low hatching rates. DNA, gamete, blood, and other detection technologies can be established to enable rapid detection of ploidy and fertility. Furthermore, maintenance and utilization of hybrid fish lineages can be supported by directional breeding and improvement of hybrid fish lineages for 4,15 , or even 30 years. The progenies can be carefully cultured in flowing water, and nets can be placed above the pools to prevent the important hybrid fish from being harmed by birds. In addition, microinjection techniques can be used to inject sperm into eggs to improve the efficiency of fertilization.

Furthermore, according to previous studies, researchers have obtained the hybrids of sheep (o) and goat $\left({ }^{*}\right)$ through mixed insemination, repeated fertilization, and miscarriage prevention measures, and the hyperthermia-inactivated sheep semen has been used to preserve goat semen. Mixed semen has been injected into a pregnant ewe to improve fertilization rates and 12 sheep-goat hybrids were obtained (Chen et al. 2018). Similarly, with regard to distant hybridization, sperm activity can be increased with inactivated semen of the same species for preservation of heterogeneous sperm.

\section{Improvement of nutrition}

Improving the nutrition of the offspring from distant hybridization is also an effective way to overcome the embryo abortion, abnormal development, and hybrid infertility. The physiological development, living habits, and nutritional needs of the hybrids are largely different from those of the parents. Therefore, if the individual's nutritional needs can be met in time to adapt to the individual's developmental characteristics, it is possible to promote the normal development of the hybrid individuals and hybrid fertility may even be able to be restored. For example, studies have shown that the addition of appropriate mannan oligosaccharides to feed can significantly improve the growth rates of the hybrids from Nile tilapia (Oreochromis niloticus) and blue tilapia (Oreochromis aureus), improving intestinal structure and non-specific immune function (Liu et al. 2009). Other studies have shown that the physiological development of yak can be promoted or restored by providing male yak with rich nutrients and increasing the amount of phosphorus in the feed (Chen et al. 2018).

\section{Establishment and application of fish lineages derived from distant hybridization}

Distant hybridization can transfer the genome of one species to another, resulting in changes in the genotypes and phenotypes of the progenies. In particular, at the phenotype level, hybridization often leads offspring to show advantages in growth rate, survival rate, productivity, feeding ability, and disease resistance. Hybridization has become an important breeding method in aquaculture. Many hybrid advantages and fish lineages derived from distant hybridization have been reported; for instance, the all-male $F_{1}$ hybrids of Nile tilapia $(\$, 2 n=44)$ and blue tilapia $(0,2 n=44)$ have strong productivity and fast growth (Wang et al. 1989), since the hybrids popularization in the 1990s, the breeding area of this fish has increased year by year and has become the main breeding species in China. And, as of around 2008, the hybrids production was about $100 \times 10^{4} \mathrm{t}$ (tons), accounting for $25 \%$ of China's tilapia production (Zhang \& Bai 2014). The reciprocal $\mathrm{F}_{1}$ hybrids of white bass (Morone chrysops, $2 \mathrm{n}=44$ ) and striped bass (Morone saxatilis, $2 \mathrm{n}=44$ ) are superior to the parents with regard to growth rate, stress resistance, and disease resistance (Gaylord \& Gatlin III 2000). In terms of fish lineages derived from distant hybridization, an $\mathrm{F}_{1}-\mathrm{F}_{3}$ lineage has been established by the hybridization of Nile tilapia (9) and Santoro tilapia (Sarotherodon melanotheron, $0,2 \mathrm{n}=44$ ) (Li et al. 2008) and an $\mathrm{F}_{1}-\mathrm{F}_{2}$ lineage has been produced from the hybridization of mandarin fish (Siniperca chuatsi, $q, 2 \mathrm{n}=48$ ) and spotted mandarin fish (Siniperca scherzeri, $0,2 \mathrm{n}=48$ ) (Yuan et al. 2014). Our team has also established a series of fish lineages with advantage traits, as described subsequently.

\section{Establishment and application of diploid lineages}

We have established some allodiploid fish lineages by hybridization of the parents with the same number of chromosomes $(2 n=100$ or $2 n=48)$. For example, a fertile hybrid lineage was established through intergeneric hybridization of female blunt snout bream $(2 n=48)$ and male topmouth culter $(2 n=48)$ and a 
new type of hybrid progeny with fast growth and high nutrition was obtained by backcrossing with $F_{1}$ hybrid (9) and blunt snout bream (o) (Xiao et al. 2014). We also established a stable hybrid lineage derived from hybridization of white crucian carp $(\%, 2 \mathrm{n}=100)$ and red crucian carp $(0,2 n=100)$ and a new type of improved fish obtained by backcrossing between $\mathrm{F}_{1}$ hybrid (o) and white crucian carp (o) (Liu et al. 2019). Furthermore, two other types of allodiploid fish lineages have been obtained from blunt snout bream $($ ( ) $) \times$ Bleeker's yellow tail (Xenocypris davidi Bleeker, $\hat{0}, 2 \mathrm{n}=48$ ) (Hu et al. 2012) and common carp (O) $\times$ red crucian carp (o). It is worth noting that these types of fish have been used for large-scale promotion and breeding, resulting in significant economic and social benefits.

In addition, we have established some types of autodiploid fish lineages. For example, an autodiploid white crucian carp lineage with a gray-white body was established derived from white crucian carp (o $2 n=100) \times$ blunt snout bream $(0,2 n=48)$ (Wang et al. 2019a). An autodiploid crucian carp-like lineage with a gray body was produced by the crossing of common carp $($ o, $2 n=100) \times$ blunt snout bream $(\hat{0}, 2 n=48)$ (Wang et al. 2017). An autodiploid red crucian carplike lineage was successfully obtained by hybridization of koi carp (C. carpio haematopterus) ( $+2 n=100)$ and blunt snout bream $(0,2 n=48)$; furthermore, a new type of goldfish-like fish with a twin tail has been created by self-crossing of the red crucian carp-like fish, which can be promoted as an ornamental fish and provides new experimental evidence for the origin of goldfish (Wang et al. 2018).

\section{Establishment and application of tetraploid lineages}

Since the 1980s, our team has carried out the intergeneric hybridization of red crucian carp $(\$, 2 n=100)$ and common carp $(0,2 n=100)$. The research results have shown that there are some bisexual fertile individuals among the $F_{1}$ hybrids and that these individuals produce $\mathrm{F}_{2}$ hybrids by self-mating. And, the bisexual fertile tetraploid $(4 n=200)$ individuals have been obtained in $\mathrm{F}_{3}$ offspring by fertilization of the unreduced eggs and the unreduced sperm produced from female and male individuals of $F_{2}$ hybrids. Now, an allotetraploid lineage $(4 n A L, 4 n=200, A A B B)\left(F_{3}-F_{26}\right)$ has been established (Fig. 3B) (Liu et al. 2001, 2016). In application, the triploid fish with fast growth and strong disease resistance have been successfully produced by crossing between diploid fish $(2 n=100)$ and allotetraploid fish and have brought significant economic benefits to aquaculture farmers (Guo et al. 2006). Triploid fish have been produced on a large scale and promoted for aquaculture in more than 20 provinces in China (Chen et al. 2009); 760 thousand tons of commercial fish were produced from 2005 to 2010, and this has been very economically profitable (Hu et al. 2012). Furthermore, the transgenic triploid fish
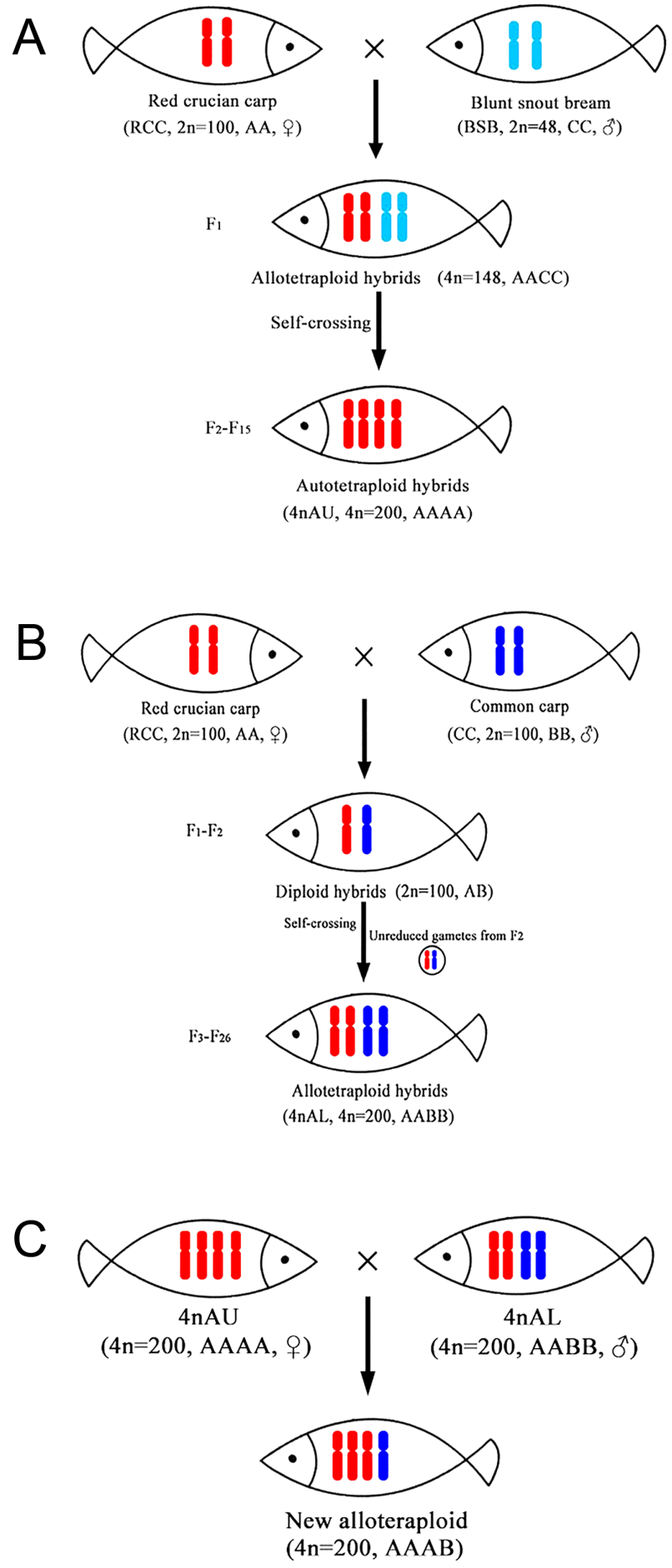

Figure 3 The formation of three tetraploid fish. (A) The formation of autotetraploid lineage ( $4 \mathrm{nAU})$; the allotetraploid hybrids were obtained by crossing of red crucian carp and blunt snout bream, and the autotetraploid hybrids was produced by self-crossing of allotetraploid hybrids. (B) The formation of allotetraploid lineage $(4 \mathrm{nAL}) ; \mathrm{F}_{1}$ hybrids were produced by hybridization of red crucian carp and common carp, and $F_{2}$ hybrids were obtained by self-mating of $F_{1}$, and $F_{2}$ can produce unreduced gametes, so the allotetraploid hybrids were obtained by self-mating of $F_{2}$. (C) A new allotetraploid fish was obtained by crossing of $4 \mathrm{nAU}$ and $4 \mathrm{nAL}$. 


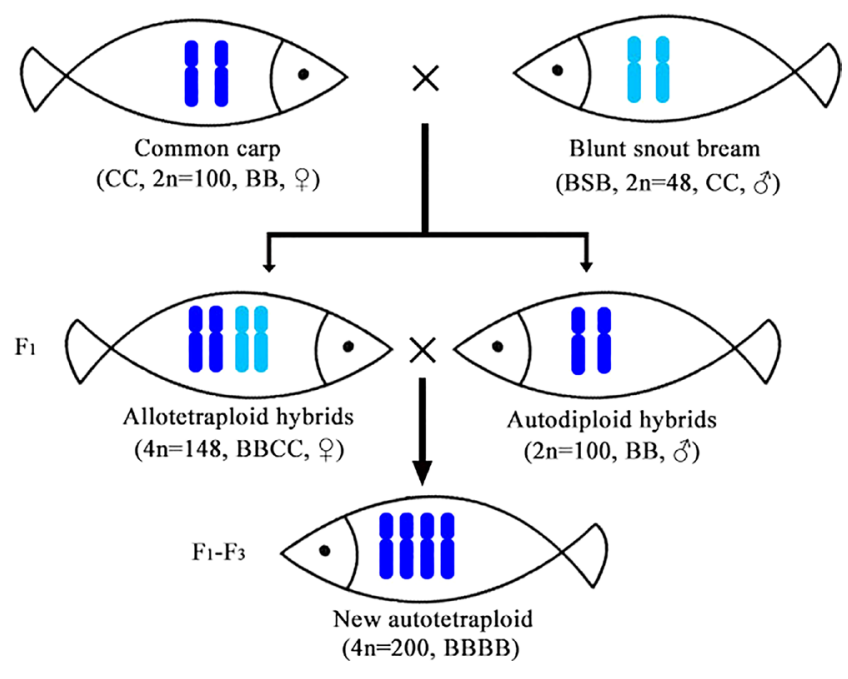

Figure 4 The formation of new autotetraploid fish. In $\mathrm{F}_{1}$ generation, allotetraploid hybrids and autoploid hybrids were produced by the hybridization of common carp and blunt snout bream, and the new autotetraploid hybrids were obtained by crossing of allotetraploid hybrids and autoploid hybrids.

can be produced by hybridization between transgenic diploid fish and allotetraploid fish. Such hybridization is an effective way to ensure the ecological safety of transgenic fish. In our previous studies, we have produced the transgenic triploid fish by crossing diploid growth hormone-transgenic yellow river carp and improved allotetraploid fish; the transgenic triploid fish have the advantages of fast growth rates, infertility, and high bait utilization (Yu et al. 2011).

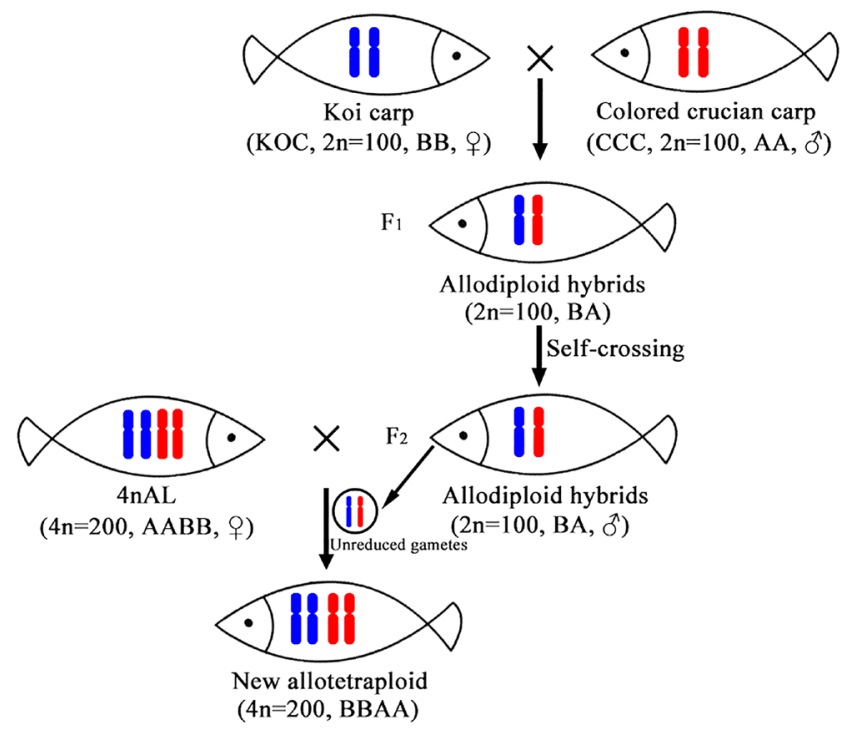

Figure 5 The formation of new allotetraploid fish. Allodiploid hybrids $\left(F_{1}\right)$ were produced by hybridization of koi carp and colored crucian carp, and $F_{2}$ hybrids (allodiploid) were obtained by self-mating of $F_{1}$, and $F_{2}$ can produce unreduced sperm. The new allotetraploid fish was obtained by crossing of $4 \mathrm{nAL}$ and $\mathrm{F}_{2}$ hybrids.
We have also obtained an autotetraploid lineage ( $4 n A U, 4 n=200, A A A A)\left(F_{2}-F_{15}\right)$ by the crossing of red crucian carp and blunt snout bream (Fig. 3A) (Qin et al. $2014 b)$. A new type of allotetraploid fish $(4 n=200$, $\mathrm{AAAB}$ ) has been obtained by the hybridization between allotetraploid fish (4nAL) and autotetraploid fish (4nAU) (Fig. 3C). In addition, we have produced the allotetraploid hybrids and autodiploid hybrids from the crossing of common carp and blunt snout bream, and a new type of autotetraploid lineage $\left(4 n=200\right.$, BBBB) $\left(F_{1}-F_{3}\right)$ was obtained by the hybridization between allotetraploid hybrids and autodiploid hybrids (Fig. 4) (Wang et al. 2019b). Furthermore, we have obtained the allodiploid hybrids $\left(F_{2}\right)$ that can produce unreduced sperm from the hybridization of koi carp and colored crucian carp; and, another new type of allotetraploid fish $(4 n=200$, BBAA) has been produced by the crossing of $4 \mathrm{nAL}$ and allodiploid hybrids (Fig. 5) (Wang et al. 2019c).

\section{Distant hybridization and Mendel's laws}

The geneticist Gregor Mendel from the Austrian empire (now the Czech Republic) presented his research results on 'Experiments in plant hybridization' at meetings of the Brunn Natural History Society on February 8th and March 8th, 1865. Through the analysis of seven pairs of traits in a hybridization experiment with peas, Mendel summarized the law of separation and the law of free combination. Mendel's laws provide an important theoretical basis for the diversity of natural species and are important guides for the practice of hybrid breeding. However, the phenomena described in Mendel's laws rarely appear during distant hybridization. In order to better understand the differences and similarities between distant hybridization and inbred hybridization and to lay the foundation for a comprehensive summary of the genetic laws of hybrid breeding, we describe the relationship between Mendel's laws and distant hybridization.

\section{Differences}

(1) The experimental basis of Mendel's laws is derived from the close hybridization of peas, which produce offspring with genetic variation. The number of parental chromosomes in Mendel's experiments is 14 and the $F_{1}$ hybrids have the same number of parental chromosomes $(2 n=14)$. However, in the distant hybridization, the parents are not closely related. The numbers of parental chromosomes can be the same or different. The progenies can have different ploidies; the $F_{1}$ hybrids can be diploid, triploid, or tetraploid. For example, gynogenetic diploid red crucian carp $(2 n=100)$, triploid hybrid fish $(3 n=124)$, and tetraploid hybrid offspring $(4 n=148)$ have been produced by the distant hybridization of red crucian carp (ㅇ, $2 \mathrm{n}=100)$ and blunt snout bream ( 0 , $2 \mathrm{n}=48)$ (Qin et al. 2014a). 
(2) The ploidy and separation results for positive and negative cross progenies in the Mendel's experiment are consistent, reflecting good parental nuclear-cytoplasmic compatibility and equal genetic contributions of the parents. However, the results are different in the offspring produced through the positive and negative crosses during distant hybridization - the parental nuclear-cytoplasmic compatibility is inconsistent and the genetic benefits of the maternal parent are obvious.

(3) The offspring of Mendel's experiment have better fertility, and the progenies produce haploid gametes. However, the fertility of progenies derived from distant hybridization is quite different (from fertile to infertile), and the offspring can produce diploid and triploid gametes in addition to haploid gametes. For instance, diploid and triploid gametes have been produced by the tetraploid fish $(4 n=148)$ obtained by the hybridization of red crucian carp and blunt snout bream (Qin et al. 2014b).

(4) In Mendel's experiment, $F_{1}$ hybrids can produce enough same types of female and male gametes because of their good fertility. After self-crossing, the gametes can be fertilized according to random combination, and the ploidy of the $F_{2}$ hybrids is consistent with that of the parents - both of them are diploid and there is a 3:1 separation phenotype ratio. However, in the $F_{1}$ hybrids of distant hybridization, due to the large difference in fertility of the progenies, the number of sperm and eggs are not enough, and the ploidy of gametes may be inconsistent. Therefore, it is difficult to produce the same kinds of gametes and obtain a phenotype ratio of 3:1 in the $F_{2}$ generation, and the ploidy of the formed $F_{2}$ individuals may also be diversified.

\section{Consistencies}

In the initial generations of offspring derived from distant hybridization (at least approximately 50 generations), the two subgenomes from the parents will change drastically and the shock effect between genomes need to be overcome. As the generations increase, the two subgenomes will tend to approach each other, become balanced and re-diploid, and finally evolve into almost the same genome, but the traces of the two subgenomes will remain. Therefore, the special characteristics of distant hybridization exist in its early generations, and it should be suitable for Mendel's laws after the genetic structure becomes stable. The formation of gametes is similar. The unreduced gametes produced in the early generations of distant hybridization result in the formation of fish with different ploidy levels. After the tetraploid and diploid lineages stabilize, Mendel's laws will also apply.

\section{Conclusions}

Hybridization is a classic breeding method. Although new techniques, such as cell engineering and genetic engineering, have received increasing attention in the field of fish breeding, these techniques are still in the stages of exploration and experimentation and are rather far from actual application - there was still a certain distance from the actual application. Close hybridization has played an important role in breeding in the past. However, the beneficial genes in fish species have been fully utilized because of the extensive development of fish resources, and the potential of close hybridization has decreased. Furthermore, the variation caused by close hybridization is far less than that of distant hybridization. Many new and greater needs of modern breeding have not been met by close hybridization. Therefore, distant hybridization is increasingly being emphasized by fish breeders worldwide. Distant hybridization can significantly expand and enrich the gene pools of fish, promote the exchange of genes between species, create new types of mutations, and even form new species. Thus, overcoming the reproductive isolations and establishing the fish lineages derived from distant hybridization are important goals for researchers. Here, we summarized some measures by which reproductive isolation can be overcome in order to establish fertile fish lineages derived from distant hybridization. We hope that this review will be helpful for fish genetic breeding. In addition, with the rapid development of genome sequencing and bioinformatics technologies, we hope that the mechanisms of reproductive isolations can be revealed thoroughly in future research. Research on fish distant hybridization is important for the fields of evolutionary biology, reproductive biology, and genetic breeding.

\section{Declaration of interest}

The authors declare that there is no conflict of interest that could be perceived as prejudicing the impartiality of this review.

\section{Funding}

This work was supported by China postdoctoral science foundation (Grant No. 2019M662789), the National Natural Science Foundation of China (Grant No. 31872551, 31730098, 31430088, 31802287, and 31802291), the earmarked fund for China Agriculture Research System (Grant No. CARS-45), the High-level Talent Agglomeration Program of Hunan (Grant No. 2019RS1044), and the Key Research and Development Program of Hunan Province (Grants No. 2018NK2072).

\section{Author contribution statement}

S J L and Q F L conceived and designed the manuscript. Q F L wrote the manuscript. J M L, L J Y, and $L \mathrm{~L}$ performed Literature collection. Q F L, S J L, M T, C Z, Q B Q, B C, M M, and C C T modified the manuscript. All authors read and approved the final manuscript. 


\section{References}

Abbott RJ 2003 Evolution. Sex, sunflowers, and speciation. Science 301 1189-1190. (https://doi.org/10.1126/science.1089292)

Anderson E \& Hubricht L 1938 Hybridization in Tradescantia. III. The evidence for introgressive hybridization. American Journal of Botany 25 396-402. (https://doi.org/10.1002/j.1537-2197.1938.tb09237.x)

Bao J \& Yan W 2012 Male germline control of transposable elements. Biology of Reproduction 86 162, 1-162,14. (https://doi.org/10.1095/ biolreprod.111.095463)

Berger F 2008 Double-fertilization, from myths to reality. Sexual Plant Reproduction 21 3-5. (https://doi.org/10.1007/s00497-007-0066-4)

Bomblies K, Lempe J, Epple P, Warthmann N, Lanz C, Dangl JL \& Weigel D 2007 Autoimmune response as a mechanism for a Dobzhansky-Mullertype incompatibility syndrome in plants. PLoS Biology 5 e236. (https:// doi.org/10.1371/journal.pbio.0050236)

Chen S, Wang J, Liu S, Qin Q, Xiao J, Duan W, Luo K, Liu J \& Liu Y 2009 Biological characteristics of an improved triploid crucian carp. Science in China: Series C, Life Sciences 52 733-738. (https://doi.org/10.1007/ s11427-009-0079-3)

Chen J, Luo M, Li S, Tao M, Ye X, Duan W, Zhang C, Qin Q, Xiao J \& Liu S 2018 A comparative study of distant hybridization in plants and animals. Science China: Life Sciences 61 285-309. (https://doi.org/10.1007/ s11427-017-9094-2)

Deng Y, Chen S, Lu A, Chen F, Tang F, Guan Z \& Teng N 2010a Production and characterisation of the intergeneric hybrids between Dendranthema morifolium and Artemisia vulgaris exhibiting enhanced resistance to chrysanthemum aphid (Macrosiphoniellasanbourni). Planta 231 693-703. (https://doi.org/10.1007/s00425-009-1081-5)

Deng Y, Teng N, Chen S, Chen F, Guan Z, Song A \& Chang Q 2010b Reproductive barriers in the intergeneric hybridization between Chrysanthemum grandiflorum (Ramat.) Kitam. and Ajania przewalskii Poljak. (Asteraceae). Euphytica 174 41-50. (https://doi.org/10.1007/ s10681-009-0113-3)

Fedoroff NV 2012 Presidential address. Transposable elements, epigenetics, and genome evolution. Science 338 758-767. (https://doi.org/10.1126/ science.338.6108.758)

Gaylord TG \& Gatlin III DM 2000 Dietary lipid level but not l-carnitine affects growth performance of hybrid striped bass (Morone chrysops $q$ $\times$ M. saxatilis ô). Aquaculture 190 237-246. (https://doi.org/10.1016/ S0044-8486(00)00404-X)

Gross BL, Schwarzbach AE \& Rieseberg LH 2003 Origin (s) of the diploid hybrid species Helianthus deserticola (Asteraceae). American Journal of Botany 90 1708-1719. (https://doi.org/10.3732/ajb.90.12.1708)

Guo X, Liu S \& Liu Y 2006 Evidence for recombination of mitochondrial DNA in triploid crucian carp. Genetics 172 1745-1749. (https://doi. org/10.1534/genetics.105.049841)

Hu J, Liu S, Xiao J, Zhou Y, You C, He W, Zhao R, Song C \& Liu Y 2012 Characteristics of diploid and triploid hybrids derived from female Megalobrama amblycephala Yih $\times$ male Xenocypris davidi Bleeker. Aquaculture 364-365 157-164. (https://doi.org/10.1016/j. aquaculture.2012.08.025)

Hu F, Fan J, Qin Q, Huo Y, Wang Y, Wu C, Liu Q, Li W, Chen X, Liu C et al. 2019 The sterility of allotriploid fish and fertility of female autotriploid fish. Frontiers in Genetics 10 377. (https://doi.org/10.3389/ fgene.2019.00377)

Karpechenko GD 1927 The production of polyploid gametes in hybrids. Hereditas 9 349-368. (https://doi.org/10.1111/j.1601-5223.1927. tb03536.x)

Levin HL \& Moran JV 2011 Dynamic interactions between transposable elements and their hosts. Nature Reviews: Genetics 12 615-627. (https:// doi.org/10.1038/nrg3030)

Li S, Yan B, Cai W, Li T, Jia J \& Zhang Y 2008 Evaluation of growth, salt tolerance and parents' heterosis contribution in reciprocal hybrids $\mathrm{F}_{2}$ between Oreochromis niloticus and Sarotherodon melanotheron. Journal of Fisheries of China 3 335-341.

Liu Y 1993 Reproductive Physiology of Chinese Cultured Fish. Beijing: Chinese Agricultural Press.

Liu S 2010 Distant hybridization leads to different ploidy fishes. Science China: Life Sciences 53 416-425. (https://doi.org/10.1007/s11427-0100057-9)
Liu S, Liu Y, Zhou G, Zhang X, Luo C, Feng H, He X, Zhu G \& Yang H 2001 The formation of tetraploid stocks of red crucian carp X common carp hybrids as an effect of interspecific hybridization. Aquaculture 192 171-186. (https://doi.org/10.1016/S0044-8486(00)00451-8)

Liu A, Leng X, Li X, Wang L \& Wan H 2009 Effect of mannan oligosaccharides on growth performance, intestinal structure and nonspecific immunity of tilapia, Oreochromis niloticus $\times$ O. aureus. Journal of Zhejiang University (Agriculture and Life Sciences) 35 329-336.

Liu S, Zhu X, Chen K, Zhao J, Li K \& Pan D 2011 Karyotype analysis of the hybrid snakehead (Channa maculata $\$ \times C$. argus ${ }^{*}$ ) and its inbred progeny $\left(\mathrm{F}_{2}\right)$. Chinese Journal of Zoology $\mathbf{4 6}$ 100-105.

Liu S, Luo J, Chai J, Ren L, Zhou Y, Huang F, Liu X, Chen Y, Zhang C, Tao M et al. 2016 Genomic incompatibilities in the diploid and tetraploid offspring of the goldfish $\times$ common carp cross. PNAS 113 1327-1332. (https://doi.org/10.1073/pnas.1512955113)

Liu Q, Qi Y, Liang Q, Xu X, Hu F, Wang J, Xiao J, Wang S, Li W, Tao M et al. 2018 The chimeric genes in the hybrid lineage of Carassius auratus cuvieri () $x$ Carassius auratus red var. (ð). Science China: Life Sciences 61 1079-1089. (https://doi.org/10.1007/s11427-017-9306-7)

Liu Q, Liu J, Liang Q, Qi Y, Tao M, Zhang C, Qin Q, Zhao R, Chen B \& Liu S 2019 A hybrid lineage derived from hybridization of Carassius cuvieri and Carassius auratus red var. and a new type of improved fish obtained by back-crossing. Aquaculture 505 173-182. (https://doi.org/10.1016/j. aquaculture.2019.02.056)

Mallet J 2007 Hybrid speciation. Nature 446 279-283. (https://doi. org/10.1038/nature05706)

Mavárez J, Salazar CA, Bermingham E, Salcedo C, Jiggins CD \& Linares M 2006 Speciation by hybridization in Heliconius butterflies. Nature 441 868-871. (https://doi.org/10.1038/nature04738)

McClure BA \& Franklin-Tong V 2006 Gametophytic self-incompatibility: understanding the cellular mechanisms involved in 'self' pollen tube inhibition. Planta 224 233-245. (https://doi.org/10.1007/s00425-0060284-2)

Melo MC, Grealy A, Brittain B, Walter GM \& Ortiz-Barrientos D 2014 Strong extrinsic reproductive isolation between parapatric populations of an Australian groundsel. New Phytologist 203 323-334. (https://doi. org/10.1111/nph.12779)

Pampoulie C, Ruzzante DE, Chosson V, Jörundsdóttir TD, Taylor L, Thorsteinsson V, Daníelsdóttir AK \& Marteinsdóttir G 2006 The genetic structure of Atlantic cod ( $G$ adus morhua) around Iceland: insight from microsatellites, the Pan I locus, and tagging experiments. Canadian Journal of Fisheries and Aquatic Sciences 63 2660-2674. (https://doi. org/10.1139/f06-150)

Qin Q, He W, Liu S, Wang J, Xiao J \& Liu Y 2010 Analysis of 5S rDNA organization and variation in polyploid hybrids from crosses of different fish subfamilies. Journal of Experimental Zoology: Part B, Molecular and Developmental Evolution 314 403-411. (https://doi.org/10.1002/ jez.b.21346)

Qin Q, Wang Y, Wang J, Dai J, Liu Y \& Liu S 2014a Abnormal chromosome behavior during meiosis in the allotetraploid of Carassius auratus red var. (o) $\times$ Megalobrama amblycephala (o). BMC Genetics 15 95. (https://doi. org/10.1186/s12863-014-0095-6)

Qin Q, Wang Y, Wang J, Dai J, Xiao J, Hu F, Luo K, Tao M, Zhang C, Liu Y et al. 2014b The autotetraploid fish derived from hybridization of Carassius auratus red var. (female) x Megalobrama amblycephala (male). Biology of Reproduction 91 93. (https://doi.org/10.1095/ biolreprod.114.122283)

Rieseberg LH \& Willis JH 2007 Plant speciation. Science 317 910-914. (https://doi.org/10.1126/science.1137729)

Rogers RL, Bedford T \& Hartl DL 2009 Formation and longevity of chimeric and duplicate genes in Drosophila melanogaster. Genetics 181 313-322. (https://doi.org/10.1534/genetics.108.091538)

Sedeek KE, Scopece G, Staedler YM, Schönenberger J, Cozzolino S, Schiestl FP \& Schlüter PM 2014 Genic rather than genome-wide differences between sexually deceptive $\mathrm{O}$ phrys orchids with different pollinators. Molecular Ecology 23 6192-6205. (https://doi.org/10.1111/ mec.12992)

Seehausen O, Butlin RK, Keller I, Wagner CE, Boughman JW, Hohenlohe PA, Peichel CL, Saetre GP, Bank C, Brännström A et al. 2014 Genomics and the origin of species. Nature Reviews: Genetics 15 176-192. (https://doi.org/10.1038/nrg3644) 
Sharma HC \& Gill BS 1983 Current status of wide hybridization in wheat. Euphytica 32 17-31. (https://doi.org/10.1007/BF00036860)

Sheppard PM 1971 Genetics of the evolutionary process. Journal of Medical Genetics 8 545-546. (https://doi.org/10.1136/jmg.8.4.545-b)

Soltis PS \& Soltis DE 2009 The role of hybridization in plant speciation. Annual Review of Plant Biology 60 561-588. (https://doi.org/10.1146/ annurev.arplant.043008.092039)

Song BH, Wang XQ, Wang XR, Sun LJ, Hong DY \& Peng PH 2002 Maternal lineages of Pinus densata, a diploid hybrid. Molecular Ecology 11 1057-1063. (https://doi.org/10.1046/j.1365-294x.2002.01502.x)

Song C, Liu S, Xiao J, He W, Zhou Y, Qin Q, Zhang C \& Liu Y 2012 Polyploid organisms. Science China: Life Sciences 55 301-311. (https:// doi.org/10.1007/s11427-012-4310-2)

Wang Z, Zhang J \& Ji G 1986 Studies on the incompatibility of fish hybridization. Acta Hydrobiologica Sinica 10 171-179.

Wang C, Xia D, Hu M \& Wang H 1989 Studies on the hybrids of (S. nilotica $\times$ S. aureao ơ) with heterosis. Freshwater Fisheries 6 14-15.

Wang HC, Chou WC, Shieh SY \& Shen CY 2006 Ataxia telangiectasia mutated and checkpoint kinase 2 regulate BRCA1 to promote the fidelity of DNA end-joining. Cancer Research 66 1391-1400. (https://doi. org/10.1158/0008-5472.CAN-05-3270)

Wang S, Ye X, Wang Y, Chen Y, Lin B, Yi Z, Mao Z, Hu F, Zhao R, Wang J et al. 2017 A new type of homodiploid fish derived from the interspecific hybridization of female common carp $\mathrm{x}$ male blunt snout bream. Scientific Reports 7 4189. (https://doi.org/10.1038/s41598-017-04582-z)

Wang Y, Yang C, Luo K, Zhang M, Qin Q, Huo Y, Song J, Tao M, Zhang C \& Liu S 2018 The formation of the goldfish-like fish derived From hybridization of female koi carp x male blunt snout bream. Frontiers in Genetics 9 437. (https://doi.org/10.3389/fgene.2018.00437)

Wang S, Tang C, Tao M, Qin Q, Zhang C, Luo K, Zhao R, Wang J, Ren L, Xiao J et al. 2019a Establishment and application of distant hybridization technology in fish. Science China: Life Sciences 62 22-45. (https://doi. org/10.1007/s11427-018-9408-x)

Wang S, Zhou P, Huang X, Liu Q, Lin B, Fu Y, Gu Q, Hu F, Luo K, Zhang C et al. $2019 \mathrm{~b}$ The establishment of an autotetraploid fish lineage produced by female allotetraploid hybrids $\times$ male homodiploid hybrids derived from Cyprinus carpio (\$) $\times$ Megalobrama amblycephala (o). Aquaculture 515 734583. (https://doi.org/10.1016/j.aquaculture.2019.734583)

Wang Y, Zhang M, Tao S, Xie X, Tan H, Cao L, Wang J, Qin Q, Zhang C, Tao M et al. 2019c Unreduced diploid sperm from diploid hybrids and formation of a new type of tetraploid hybrid. Aquaculture $\mathbf{5 1 5} 734584$ (https://doi.org/10.1016/j.aquaculture.2019.734584)

Whitt GS 1981 Developmental genetics of fishes: isozymic analyses of differential gene expression. American Zoologist 21 549-572. (https:// doi.org/10.1093/icb/21.2.549)
Wolff K \& Peters-van Rijn J 1993 Rapid detection of genetic variability in chrysanthemum (Dendranthema grandiflora Tzvelev) using random primers. Heredity 71 335-341. (https://doi.org/10.1038/hdy.1993.147)

Wu CI \& Ting CT 2004 Genes and speciation. Nature Reviews: Genetics 5 114-122. (https://doi.org/10.1038/nrg1269)

Xiao J, Kang X, Xie L, Qin Q, He Z, Hu F, Zhang C, Zhao R, Wang J, Luo K et al. 2014 The fertility of the hybrid lineage derived from female Megalobrama amblycephala $x$ male Culter Alburnus. Animal Reproduction Science 151 61-70. (https://doi.org/10.1016/j. anireprosci.2014.09.012)

Xu M \& Shaw KL 2019 The genetics of mating Song evolution underlying rapid speciation: linking quantitative variation to candidate genes for behavioral isolation. Genetics 211 1089-1104. (https://doi.org/10.1534/ genetics.118.301706)

Xu S, Schlüter PM \& Schiestl FP 2012 Pollinator-driven speciation in sexually deceptive orchids. International Journal of Ecology 2012 1-9. (https://doi.org/10.1155/2012/285081)

Ye Y, Wu Q \& Chen R 1989 Studies on cytology or crosses between grass carp and carp-asynchronization between nucleus and cytoplasm in distant hybridization of fishes. Acta Hydrobiologica Sinica 3 234-239.

Ye L, Jiao N, Tang X, Chen Y, Ye X, Ren L, Hu F, Wang S, Wen M, Zhang C et al. 2017 Chimeras linked to tandem repeats and transposable elements in tetraploid hybrid fish. Marine Biotechnology 19 401-409. (https://doi. org/10.1007/s10126-017-9764-6)

Yu F, Xiao J, Liang X, Liu S, Zhou G, Luo K, Liu Y, Hu W, Wang Y \& Zhu Z 2011 Rapid growth and sterility of growth hormone gene transgenic triploid carp. Chinese Science Bulletin 56 1679-1684. (https://doi. org/10.1007/s11434-011-4446-7)

Yuan Y, Liang X, Tian C, Yan W, Cai W, Dou Y \& Yi T 2014 Identification of embryonic development hybrids F1 of Siniperca chuatsi ( $($ ) $\times$ Siniperca schezeri ( $\left.{ }^{\star}\right)$ and its F2. Hubei Agricultural Sciences 53 4920-4923.

Zhang L \& Bai J 2014 The main fish hybrids and current situation of aquaculture in China. Marine Fisheries 36 475-480.

Zhang S, Zhang G \& Li L 2012 Reproductive isolation of interspecific crosses between Argopecten irradians and Argopecten purpuratus. Marine Sciences 36 9-14.

Received 3 December 2019

First decision 23 January 2020

Revised manuscript received 26 February 2020

Accepted 19 March 2020 